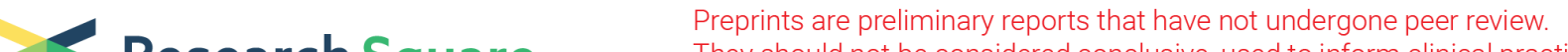 Research Square They should not be considered conclusive, used to inform clinical practice, or referenced by the media as validated information.
}

\section{Upregulated circRNA_100269 suppresses the growth and metastasis of gastric cancer by inactivating PI3K/Akt signaling pathway}

zhongli wang

First Affiliated Hospital of Jinzhou Medical University

chao liu ( $\square$ chao_liu15@163.com)

First Affiliated Hospital of Jinzhou Medical University https://orcid.org/0000-0003-4609-6899

\section{Primary research}

Keywords: circRNA_100269, gastric cancer, PI3K/Akt signaling, growth, metastasis

Posted Date: June 24th, 2020

DOl: https://doi.org/10.21203/rs.3.rs-21351/v3

License: (c) (1) This work is licensed under a Creative Commons Attribution 4.0 International License.

Read Full License 


\section{Abstract}

Background: The expression of circRNA_100269 in gastric cancer (GC) tissues and cells, together with its regulatory roles on GC cells were investigated.

Methods: The levels of circRNA_100269 in GC and matched para-carcinoma tissues, as well as in human GC cell lines and normal gastric epithelial cells were evaluated using RT-qPCR. The models with overexpression or knockdown of circRNA_100269 were generated using lentiviral vectors. Cell viability was examined using MTT assay; cell migration and invasive activity were determined by wound healing and Transwell assay. Cell cycle arrest and apoptosis were assessed; molecules involved in PI3K/Akt signaling, apoptosis and EMT were evaluated using RT-qPCR and immunoblotting. Tumour growth and expression of relevant proteins were examined in circRNA_100269 knockout mice.

Results: The results indicated the expression of circRNA_100269 was dramatically decreased in GC samples compared with para-carcinoma tissues $(p<0.05)$, while the levels of PI3K were notably increased $(p<0.05)$. Moreover, the level of circRNA_100269 was relevant to histology grade and occurrence of metastasis in GC patients $(p<0.05)$, where circRNA_100269 and PI3K was inversely correlated $(p<0.05)$. Additionally, circRNA_100269 was downregulated in GC cells compared with normal gastric epithelial cells. Overexpressed circRNA_100269 remarkably suppressed the proliferation, migration, invasion and EMT of GC cells $(p<0.05)$, induced cell cycle arrest at G0/G1 phase and promoted cell apoptosis $(p<0.05)$. In addition, PI3K/Akt signaling was involved in circRNA_100269-mediated proliferation, migration, invasion, EMT and apoptosis in GC cells ( $p<0.05)$. Knockdown of circRNA_100269 also significantly promoted tumor growth in vivo $(\mathrm{p}<0.05)$.

Conclusions: the data of the present study suggested that the expression level of circRNA_100269 was decreased in GC tissues and cells. In addition, circRNA_100269 inhibited the progression of GC by suppressing PI3K/Akt signaling. Therefore, circRNA_100269/PI3K/Akt axis may be a potential therapeutic target for GC treatment.

\section{Background}

Gastric cancer (GC) is a type of aggressive tumor and one leading cause of cancer-related mortality, and the incidence of GC are rising worldwide (1). The pathogenesis of GC is complex, and previous study has revealed that the five-year survival rate of GC patients in China remains poor ( 25-30\%; 2$)$. Recently, therapeutic approaches for GC have been developed and endoscopic/surgical resection is broadly used for patients with GC; however, the prognosis of GC is still poor. The therapeutic outcome of patients with $\mathrm{GC}$ is relevant to disease staging at diagnosis, and the mortality of patients with advanced GC remains high ( 30-50\%; 3-5). Nevertheless, the molecular mechanisms during the onset and development of GC are still unclear. Thus, it is essential to identify putative diagnostic and prognostic biomarkers for this disease. 
Circular RNAs (circRNAs) are a group of new non-coding RNAs. Not like their linear counterparts, they can form a continuous circle which is characterized with a more stable structure (6-8). Due to the absence of free 5'- or 3'- overhangs in the structure, circRNAs are resistant to the degradation caused by exonuclease (6). Furthermore, the distributions of circRNAs are tissue specific (8). Some circRNAs have been revealed as putative gene regulators, the regulatory functions of most circRNAs are not fully understood (9). Recent studies have revealed that circRNAs could be able to exert their function as miRNA 'sponges' which competitively suppress the activity of corresponding miRNAs (10). In addition, circRNAs are involved in the initiation and development of numerous diseases, including nervous system disorders and tumor (11-13). For instance, previous research has revealed the regulatory functions of a novel circRNA SFMBT2 in GC, and it could act as a sponge for miR-182-5 p to regulate the growth of tumor (14). Furthermore, recent report revealed downregulation of circRNA_100269 in GC, which inhibits tumor growth through regulating miR-630 (15). However, the detailed functions of circRNA_100269 have not been reported. Therefore, the regulatory roles and novel molecular targets of circRNA_100269 in GC were still elusive and require further elucidation.

Previous research suggested that miR-630 is associated with the regulation of various biological processes including EMT through numerous pathways such as PI3K/Akt signaling $(16,17)$. PI3K can be activated by oncogenes, consequently activated PI3K is capable of promoting the growth and metastasis of tumor cells (18). Akt is a downstream molecule of PI3K, and it is associated with numerous biological processes, such as cell proliferation, invasion and EMT (19). The activation of Akt could induce the migration, invasion and EMT of cancer cells (20). Activation of PI3K/Akt could contribute to upregulated migratory/invasive abilities of human osteosarcoma cells (21). Therefore, inhibition of PI3K/Akt pathway could be a novel therapeutic strategy for cancer treatment. In our study, the effects of circRNA_100269modulated PI3K/Akt signaling on the growth and metastasis of GC cells were investigated. The data suggested that cell cycle was arrested at G0/G1 phase and cell apoptosis was promoted in GC cells overexpressing circRNA_100269, where the levels of p-Akt and Bcl-2 were also notably reduced. These data suggested that overexpressed circRNA_100269 could inhibit the proliferation, migration and invasion while inducing the cell cycle arrest and apoptosis of GC cells via PI3K/Akt signaling. Our data could provide novel insight on the treatments of GC.

\section{Materials And Methods}

Clinical specimens. A total of 56 paired GC and non-cancerous tissues ( $\geq 5 \mathrm{~cm}$ from tumor margin; 26 males and 30 females; age range: 40-78 years old) were obtained during surgery at the First Affiliated Hospital of Jinzhou Medical University (Jinzhou, China) during July 2010 and June 2013. The tissues were cut into sections and snap-frozen using liquid nitrogen immediately after surgery, and then stored at $-80^{\circ} \mathrm{C}$ until further use. The patients were sorted into circRNA_100269 high- or low-expression groups according to average circRNA_100269 level. All the biopsies were checked by two independent pathologists, and clinicopathological features of enrolled patients were presented in Table I. Overall survival rates were evaluated using Kaplan-Meier method. Written informed consents were signed by 
patients, and all the tissues were kept anonymously. The present study was approved by the Medical Ethics Committee of the First Affiliated Hospital of Jinzhou Medical University.

Immunohistochemistry analysis. Immunostaining of phospho-PI3K was performed on paraffin-embedded sections. The biopsies were dewaxed using xylene and rehydrated through a graded ethanol series and water. Antigen retrieval was carried out using microwave treatment in $10 \mathrm{mM}$ sodium citrate for $15 \mathrm{~min}$. Tissues were then incubated with FCS at room temperature for 30 min and with primary antibody against phospho-PI3K (1:200; cat. no. ab182651, Abcam) in a humid chamber at $4^{\circ} \mathrm{C}$ overnight. The following day, the sections were rinsed in TBS for three times and incubated with biotinylated secondary antibody (1:100; Dako, UK). Antigen was then detected using a streptavidin-biotin-peroxidase system (ABC kti; Dako, UK). Staining intensity was quantified using Image J software (version 1.46; NIH, Bethesda, MD, USA).

Cell culture. Four human GC cell lines (AGS, SGC-7901, BGC-823 and MKN-45) as well as one normal human gastric epithelial cell line (GES-1) were obtained from the American Type Culture Collection (Manassas, VA, USA). The cells were maintained within DMEM supplemented with $10 \%$ fetal bovine serum (FBS; GE Healthcare Life Science), $100 \mu \mathrm{g} / \mathrm{ml}$ streptomycin and $100 \mathrm{U} / \mathrm{ml}$ penicillin (HyClone; GE Healthcare Life Science), and kept at $37^{\circ} \mathrm{C}$ in a humid incubator supplied with $5 \% \mathrm{CO}_{2}$.

Cell transfection. To establish cell model overexpressing circRNA_100269, wildtype (o/e-circRNA_100269) or mutant (o/e-NC) circRNA_100269 fragment was amplified by PCR, and subsequently integrated into pcDNA3.1 vector (Invitrogen; Thermo Fisher Scientific, Inc., Waltham, MA, USA). In order to produce circRNA_100269 knockdown model, shRNA against circRNA_100269 (sh-circRNA_100269) or negative control (sh-NC) were purchased from Genepharm Co. Ltd. (Shanghai, China). Following annealing, shRNA were inserted into lentiviral pU6-Luc-Puro vector (Genepharm Co. Ltd.). Cells without any shRNA treatment were used as the control group. Up- or down-regulation of circRNA_100269 was further evaluated by RTqPCR. All the transfections were conducted using Lipofectamine ${ }^{\circledR} 2000$ (Invitrogen; Thermo Fisher Scientific, Inc.). Eight hours following transfection, culture supernatants were replenished by fresh DMEM containing 10\% FBS. For the inhibition of PI3K signalling, cells were treated with LY294002 (10mM; Cell Signaling Technology, Beverly, USA).

$R N A$ extraction and reverse transcription-quantitative polymerase chain reaction ( $R T-q P C R)$. Total RNA from clinical specimens or cells was isolated using TRIzol ${ }^{\circledR}$ reagent (Invitrogen; Thermo Fisher Scientific, Inc.), and then reverse transcribed into cDNA by a PrimeScript ${ }^{\text {Tt" }}$ RT kit (Takara Biotechnology Co., Ltd., 
Dalian, China). The target cDNA was amplified using SYBR Green PCR Master Mix (TaKaRa Biotechnology Co., Ltd.), and the reaction was carried out on an ABI 7500 Real-Time PCR system (Thermo Fisher Scientific, Inc.) Endogenous GAPDH was used as internal control. The sequences of forward and reverse primer were as follows: circRNA_100269, 5'-CTATGTCGTTCAAGGAAGA-3' and 5'GTCTTGAGACTGGGTATGT-3'; PI3K, 5'-AACACAGAAGACCAATACTC-3' and 5'-TTCGCCATCTACCACTAC-3'; E-cad, 5'-AAGAAGCTGGCTGACATGTACGGA-3' and 5'-CCACCAGCAACGTGATTTCTGCAT-3'; vimentin, 5'AGAACCTGCAGGAGGCAGAAGAAT-3' and 5'-TTCCATTTCACGCATCTGGCGTT-3'; Snail, 5'TTTCTGGTTCTGTGTCCTCTGCCT-3' and 5'-TGAGTCTGTCAGCCTTTGTCCTGT-3'; GAPDH, 5'GCAAGAGCACAAGAGGAAGA-3' and 5'-ACTGTGAGGAGGGGAGATTC-3'. PCR program was $95^{\circ} \mathrm{C}$ for 5 min, followed by 45 cycles of $95^{\circ} \mathrm{C}$ for $15 \mathrm{~s}, 60^{\circ} \mathrm{C}$ for $20 \mathrm{~s}$ and $72^{\circ} \mathrm{C}$ for $10 \mathrm{~s}$. Relative expression levels were analyzed using $2^{-\Delta \Delta \mathrm{Cq}}$ method.

Western blotting. Total protein was extracted by radioimmunoprecipitation buffer (Beyotime Institute of Biotechnology, Shanghai, China). The concentration of isolated protein was measured by bicinchoninic acid assay (Beyotime Institute of Biotechnology). Equal amount $(30 \mu \mathrm{g})$ of protein samples were loaded onto SDS-PAGE gel and then transferred onto a PVDF membrane (EMD Millipore, Billerica, MA, USA). Membranes were subsequently blocked with tris-buffered saline (TBS) supplemented with $5 \%$ skimmed milk at room temperature for $2 \mathrm{~h}$ and then incubated in corresponding primary antibodies as follows: PI3K (1:2000; cat. no. ab140307; Abcam), Akt (1:1000; cat. no. 9272; Cell Signaling Technology), p$\mathrm{Akt}^{\mathrm{S473}}$ (1:1000; cat. no. 4058; Cell Signaling Technology), p53 (1:1000, cat. no. 9282; Cell Signaling Technology), Bcl-2 (1:1000, cat. no. 15071; Cell Signaling Technology), cyclin D1 (1:2000, cat. no. 2926; Cell Signaling Technology), E-cad (1:1000, cat. no. 3195; Cell Signaling Technology), vimentin (1:2000; cat. no. 5741; Cell Signaling Technology), snail (1:1000; cat. no. 3879; Cell Signaling Technology), Bax (1:1000; cat. no. 2772; Cell Signaling Technology), cas-9 (1:2000; cat. no. 14697; Cell Signaling Technology), cleaved cas-3 (1:1000; cat. no. 9661; Cell Signaling Technology), cleaved PARP (1:1000; cat. no. 9541; Cell Signaling Technology) or GAPDH (1:1,000; cat. no. sc-47724; Santa Cruz Biotechnology Inc.) at $4^{\circ} \mathrm{C}$ overnight. The following day, membranes were incubated in horseradish peroxidase-labelled anti-mouse (1:5,000; cat. no. sc-2371; Santa Cruz Biotechnology Inc.) or -rabbit IgG (1:5000; cat. no. sc2357; Santa Cruz Biotechnology Inc.) at room temperature for $1 \mathrm{~h}$. Protein bands were visualized using ECL protein detection reagent (Pierce Biotechnology; Thermo Fisher Scientific, Inc). Signals were quantified by densitometric method using Image $\mathrm{J}$ software (version 1.46; NIH, Bethesda, MD, USA).

Cell proliferation assay. Cells were harvested $24 \mathrm{~h}$ post-transfection, and a total of $1 \times 10^{4}$ cells were placed in each well of a 96-well plate. The proliferation of cells was evaluated by MTT assay (SigmaAldrich; Merck KGaA) at day 1, 2, 3 and 4. Briefly, $20 \mu$ of MTT solution was added into the cells, and the plates were incubated at $37^{\circ} \mathrm{C}$ for $4 \mathrm{~h}$, the absorbance at $570 \mathrm{~nm}$ was measured using a microplate reader (Bio-Rad Laboratories, Inc., Hercules, CA, USA). 
Wound healing assay. Cells were seeded on a 6-well plate at a density of $4 \times 10^{5}$ cells/well and transfected with corresponding plasmids. After the cells reached a confluency of $\sim 80-100 \%$, they were pre-treated with $10 \mathrm{ug} / \mathrm{mL}$ mitomycin $\mathrm{C}$ (Thermo Fisher Scientific) for two hours prior to wound healing assay. Then, cell monolayer was scratched by a straight line with a sterile micropipette tip and rinsed three times with PBS, which was replaced with fresh DMEM. Subsequently, the scratch width changes were monitored immediately following the scratch was formed and after 6,12 and $24 \mathrm{~h}$. The images were captured using a fluorescence microscope (magnificationx 100 , Olympus Corporation, Tokyo, Japan). The migration of cells was analysed by Image J using the following formula: Migration area ratio = proportion of closed wound area/entire field of view area.

Transwell assay. Cells were pre-treated with $10 \mathrm{ug} / \mathrm{mL}$ mitomycin C (Thermo Fisher Scientific) for two hours prior to assay. A total of $1 \times 10^{5}$ cells were diluted in FBS-free culture media and seeded onto the upper chamber (BD Biosciences, Franklin Lakes, New Jersey, USA) that is pre-coated with Matrigel ${ }^{\circledR}$ (Sigma-Aldrich, St. Louis, MO, USA). Subsequently, $500 \mu$ l culture media containing $10 \%$ FBS was added into the lower chamber. Following overnight incubation, non-invasive cells were detached by a cotton swab, whereas invaded cells in the lower chamber were fixed using $4 \%$ paraformaldehyde and stained in $0.5 \%$ crystal violet. The numbers of invasive cells were counted in five randomly selected fields using an inverted light microscope (magnificationx200, Olympus Corporation, Tokyo, Japan).

Cell cycle and apoptotic rate analysis. Cells were inoculated onto a 6-well plate with a density of $4 \times 10^{5}$ cells/well following the treatments with o/e-circRNA_100269 or o/e-NC, respectively. Subsequently, cells were collected using low-speed centrifugation (1000rpm) at $4^{\circ} \mathrm{C}$ for 5 mins. Cell pellets were rinsed and resuspended using PBS, subsequently fixed in $70 \%$ pre-chilled ethanol and kept at $4^{\circ} \mathrm{C}$ for two days. Cells were lysed prior to flow cytometry, an then centrifuged and re-suspended using propidium iodide $(\mathrm{PI}$, Sigma-Aldrich, USA) staining buffer containing $50 \mu \mathrm{l} / \mathrm{ml}$ of PI as well as $250 \mu \mathrm{l} / \mathrm{ml}$ RNase A. The distribution of cell cycle was determined by a flow cytometer (BD Biosciences, USA) and then analysed by Flowjo version 7.6 software (Flowjo LLC, USA). In order to evaluate cell apoptosis, cell suspension was incubated with PI in dark at $4^{\circ} \mathrm{C}$ for 30 mins and then stained by $5 \mu$ annexin V-FITC (JingMei Biotech, Beijing, China). Apoptotic rate was examined using flow cytometer (BD Biosciences, USA) and then interpreted by Flowjo version 7.6 software (Flowjo LLC, USA).

In vivo nude mouse xenograft. Female BALB/C nude mice ( $\sim 5$ weeks old) with the weight of $\sim 19 \mathrm{~g}$ were obtained from The Laboratory Animal Research Centre of Nantong University (Nantong, China). The mice were routinely housed in a temperature- $\left(22 \pm 2^{\circ} \mathrm{C}\right)$ and humidity-controlled $(\sim 60 \%)$ environment, under a 
12-h dark/light cycle with libitum access to food and water for more than three days before the experiments. Mice were randomly grouped ( $n=5$ in each group) and injected with AGS cells transfected with sh-NC or sh-circRNA_100269. Briefly, a total of $1 \times 10^{7}$ cells were diluted in $200 \mu \mathrm{l}$ PBS and then injected into the back of mice subcutaneously. Mice with developing tumors were closely monitored four times a week. Six weeks following injection, the mice were sacrificed. Tumor tissues were removed and evaluated. Tumor volume was calculated as follows: $V\left(\mathrm{~mm}^{3}\right)=\left(\right.$ length $\mathrm{x}$ width$\left.{ }^{2}\right) / 2$. To trigger the metastasis, $1 \times 10^{5}$ of cells were suspended using $20 \mu \mathrm{l}$ PBS and subsequently injected into the lateral tail vein of mice. Experiments contained 10 mice inoculated with tumor cells on day 0 . Post-injection, mice were randomly sorted into experimental groups and then evaluated 42 days later. The protocols of animal experiments were approved by the Ethics Committee of our Hospital. The animal work was carried out in accordance with the National Institutes of Health Guide for the Care and Use of Laboratory Animals.

Statistical analysis. Data were presented as means \pm standard error of mean and interpreted using SPSS 17.0 (SPSS, Inc., Chicago, IL, USA). The significance of differences was determined by one-way analysis of variance (ANOVA) or the Student's t-test. A student-Newman-Keuls test was conducted following ANOVA. The associations between RNA expression levels were evaluated by Pearson's correlation analysis. $P<0.05$ was considered to indicate a statistically significant difference.

\section{Results}

The expression of circRNA_100269 is reduced in GC tissues and cells. The expression levels of circRNA_100269 were determined in 56 paired GC and non-tumor tissues using RT-qPCR. The data suggested that circRNA_100269 was significantly downregulated in GC samples compared to paracarcinoma controls (Fig. 1A). Additionally, the relationship between circRNA_100269 and the development of GC was evaluated, and the results indicated that circRNA_100269 was notably reduced in patients with advanced GC (Fig. 1B). Additionally, the expression of circRNA_100269 was remarkably downregulated in GC patients with metastasis (Fig. 1C). Moreover, GC patients with relatively low levels of circRNA_100269 exhibited significantly reduced overall survival ( $p=0.0025$, log-rank test; Fig. 1D). Similarly, downregulation of circRNA_100269 was also detected in GC cells comparing to normal gastric epithelial cells (Fig.1E). Taken all together, the level of circRNA_100269 was decreased in GC, which may lead to the progression of tumor.

Overexpression of circRNA_100269 suppresses the growth, metastasis and EMT of GC cells. In order to investigate the effects of circRNA_100269 on the progression of GC, circRNA_100269 was overexpressed in AGS and MKN-45 cells. The transfection efficiencies were evaluated by RT-qPCR (Fig. 2A). Furthermore, the data of MTT assay revealed that the viabilities of GC cells transfected with o/e-circRNA_100269 was suppressed (Fig. 2B and C). Would healing assay was performed to evaluate cell migration, whereas 
invasive activity was determined using Transwell assay. Our data revealed that cell migration/invasion was inhibited within the circRNA_100269 overexpression group compared with the control (Fig. 2D-G). To investigate the effects of overexpressed circRNA_100269 on EMT of GC cells, the protein and mRNA levels of associated molecules such as E-cad, vimentin and snail were evaluated. The expression levels of abovementioned proteins were affected in cells transfected with o/e- circRNA_100269 (Fig. 2H and I). In summary, overexpressed circRNA_100269 may result in suppressed proliferation, migration, invasion and EMT of GC cells.

Overexpressed circRNA_100269 promotes cell cycle arrest and apoptosis in GC cells. According to the abovementioned results, circRNA_100269 was able to affect the proliferation and metastasis of GC cells. Furthermore, to investigate the influences of overexpressed circRNA_100269, the distribution of cell cycle and apoptosis in GC cells transfected with o/e-circRNA_100269 were evaluated compared with the control. The results revealed that GC cell cycle was dramatically shifted from $S$ and G2/M phase to G0/G1 phase, and the cell percentage in G0/G1 phase was significantly increased, whereas those in $S$ phase was notably reduced (Fig. $3 \mathrm{~A}$ and B). Furthermore, the results of flow cytometry revealed that overexpressed circRNA_100269 also promoted the apoptosis of GC cells (Fig. 3C and D), which was confirmed by the upregulation of apoptosis-related markers including Bax, cleaved cas-3 and PARP (Fig. 3E). Our findings suggested that upregulation of circRNA_100269 could arrest cell cycle in G0/G1 phase to induce cell apoptosis.

Knockdown of circRNA_100269 induces the development of GC cells. As part of gain- and loss-offunction study, circRNA_100269 was knockdown in GC cells. The efficiencies of shRNA were confirmed by RT-qPCR (Fig. 4A). MTT assay indicated that the cell viabilities were increased following the transfection with sh-circRNA_100269 (Fig. 4B and C). The results of wound healing and Transwell assays revealed that cell migrating and invasive abilities were enhanced in GC cells transfected with shcircRNA_100269 (Fig. 4D-G). Furthermore, the levels of EMT-related molecules were influenced in shcircRNA_100269-transfected group (Fig. 4H and I). Taken all together, knockdown of circRNA_100269 could lead to enhanced proliferation, migration, invasion and EMT within GC cells.

Knockdown of circRNA_100269 suppressed cell cycle arrest and apoptosis in GC cells. To study the effects of downregulated circRNA_100269 on cell cycle distribution and apoptosis, further experiments were performed. The results suggested that cell percentage in G0/G1 phase was remarkably reduced, whereas that in S phase was notably increased (Fig. 5A and B). Additionally, the results of flow cytometry indicated that downregulated circRNA_100269 expression inhibited the apoptosis of GC cells (Fig. 5C and D), which was also confirmed by the downregulation of apoptosis-associated markers (Fig. 5E). In 
summary, these findings revealed that knockdown of circRNA_100269 could inhibit cell cycle arrest and apoptosis in GC.

PI3K/Akt signaling pathway is the novel target of circRNA_100269 in GC. Further experiments were conducted to investigate the mechanisms of circRNA_100269-modulated cell growth and apoptosis in GC. Western blot analysis indicated that overexpressed circRNA_100269 was able to downregulate the expression levels of PI3K and phosphorylated Akt (p-Akt), while knockdown of circRNA_100269 enhanced the expression of PI3K and p-Akt (Fig. 6A). Moreover, the downstream targets of PI3K/Akt signaling such as p53, Bcl-2 and cyclin D1 were examined. As presented in western blotting, the protein levels of Bcl-2 and cyclin D1 were decreased, whereas p53 was increased in GC cells overexpressing circRNA_100269, and vice versa. In addition, the levels of PI3K were evaluated in GC and para-carcinoma tissues. The results revealed significant upregulation of PI3K in GC samples compared with normal controls (Fig. 6B). Moreover, the expression of PI3K was notably increased in patients with advanced GC (Fig. 6C), and the level of PI3K was remarkably upregulated in GC patients with metastasis (Fig. 6D). Additionally, the expression levels of PI3K and circRNA_100269 were inversely correlated in GC patients (Fig. 6E), where the activity of PI3K and the expression of circRNA_100269 were also negatively correlated (Fig. 6F). Furthermore, the level of PI3K was notably elevated in GC cell lines (Fig.6G). Our data indicated that circRNA_100269 could affect the development of GC by regulating PI3K/Akt pathway.

Inhibition of PI3K/Akt signaling reverses the biological behavior changes caused by circRNA_100269 in GC cells. To study whether the influences of circRNA_100269 on the progression of GC cells was modulated through PI3K/Akt pathway, GC cells were transfected by sh-NC, sh-circRNA_100269 or cotreated with LY294002. The results indicated that the effects caused by downregulated circRNA_100269 in GC cells were abolished by inactivation of PI3K/Akt signaling (Fig. 7). These findings revealed that PI3K/Akt signaling is involved in circRNA_100269-mediated cell growth and metastasis in GC, suggesting circRNA_100269 could suppress the development of GC by suppressing PI3K/Akt pathway.

Knockdown of circRNA_100269 promotes the progression of GC in vivo. To study if sh-circRNA_100269 promotes the development of GC, cells transfected with sh-circRNA_100269 or the control were injected in $\mathrm{BALB} / \mathrm{C}$ nude mice subcutaneously. Six weeks following the inoculation, mice were sacrificed. Tumor tissues were isolated and examined (Fig. 8A). The average tumor volume of sh-circRNA_100269 group was notably increased (Fig. 8B). In addition, the tumor weight of circRNA_100269 knockout mice was significantly elevated (Fig. 8C). Additionally, the numbers of macroscopic nodules were remarkably elevated in sh-circRNA_100269 mice (Fig. 8D). Moreover, the results of western blot analysis suggested that EMT-, apoptosis- and PI3K/Akt-associated markers exhibited the same expression pattern as 
observed in the in vitro assays (Fig. 8E). The results suggested that knockdown of circRNA_100269 could promote the development of $\mathrm{GC}$ by inhibiting $\mathrm{PI} 3 \mathrm{~K} /$ Akt signaling in vivo.

\section{Discussion}

GC is a common type of malignancy that is a major cause of cancer-related mortality $(1,2)$. Although the recent treatments of GC have been developed, the therapeutic outcome of this disease still remains poor (3-5). Accumulating evidences have suggested that circRNAs are associated with tumorigenesis of numerous types of cancer (11-13). Furthermore, aberrant levels of circRNAs has been found in tumor cells, indicating the potential roles of circRNAs during the development of cancer including GC. Among these IncRNAs, circRNA_100269 was downregulated in GC, which functions as tumor suppressor through regulating miR-630 (15). Furthermore, miR-630 serves essential roles during EMT via various pathways including PI3K/Akt signaling $(16,17)$. However, the exact functions and downstream molecular targets of circRNA_100269 in GC were not completely understood and require further investigation. Thus, our findings could provide novel insight on the therapeutic development of GC. The activation of PI3K could be triggered by oncogenes, consequently resulting in the development of tumor (18). Akt is a downstream molecule of $\mathrm{PI} 3 \mathrm{~K}$, which is associated with cell growth and EMT, and activated Akt could promote the migration, invasion and EMT of cancer cells such as osteosarcoma cells (19-21). Therefore, inactivation of $\mathrm{PI3K} /$ Akt signaling could be a putative therapeutic strategy for cancer treatment.

In this study, the effects of circRNA_100269-modulated PI3K/Akt axis on the growth and metastasis of GC were elucidated. Our results revealed significant downregulation of circRNA_100269 in GC patients, whereas the levels of PI3K were remarkably elevated, and their expression were correlated with the tumor grading and occurrence of metastasis. In addition, downregulated circRNA_100269 and upregulated PI3K were detected in GC cells. Overexpressed circRNA_100269 notably inhibited the growth, metastasis and EMT of GC cells, and vice versa. Furthermore, our data also suggested cell cycle was arrested at G0/G1 phase and cell apoptosis was promoted in GC cells overexpressing circRNA_100269, where the expression levels of PI3K/Akt-associated molecules were also affected, suggesting the biological behavior changes in GC cells caused by circRNA_100269 could be dependent on PI3K/Akt signaling. Knockdown of circRNA_100269 significantly promoted tumor development both in vitro and in vivo. These data suggested that circRNA_100269 could inhibit the proliferation, migration and invasion while inducing the cell cycle arrest and apoptosis of GC cells by suppressing PI3K/Akt signaling pathway. In consistence with our findings, recent studies also reported circRNAs including circNHSL1 and circNF1 could be novel gene regulators in $\mathrm{GC}$, which can affect tumor progression through various signaling pathways (22-27). Taken all together, our results revealed the essential roles of circRNA_100269/PI3K/Akt signaling on the modulation of GC progression.

\section{Conclusion}

In summary, our results suggested that circRNA_100269 was a promising tumor suppressor that could inhibit the development of GC by targeting PI3K/Akt pathway. However, there were some limitations in the 
present study, for instance, to confirm the findings of MTT assay, the expression levels of proliferationrelated molecules such as PCNA could be evaluated; additionally, to validate the data of Transwell assay, the levels of invasion-associated markers including cathepsin and uPA may also be determined; furthermore, to confirm whether circ_RNA100269 is a biomarker for aggressive primary and metastatic GC, the expression profiles of circ_RNA100269 and its downstream molecules should also be evaluated in larger sample size. Our data revealed the key functions of circRNA_100269 and the potential mechanisms underlying the growth and metastasis of GC, which provided substantial evidences on the roles of circRNA_100269 in tumorigenesis. Therefore, circRNA_100269 could be a novel marker for the diagnosis and treatment for GC. At present, inhibition of PI3K/Akt signaling is considered as a potential therapeutic approach in cancer treatment (28-33). CircRNA_100269/PI3K/Akt axis could be a putative therapeutic target for the treatment of GC.

\section{Abbreviations}

CircRNAs: circular RNAs; GC: gastric cancer.

\section{Declarations}

\section{Acknowledgements}

Not applicable.

\section{Funding}

This study was supported by the Natural Science Foundation of Liaoning Province (grant no. 20170540401).

\section{Availability of data and materials}

The datasets generated in our present study are included in this published article.

\section{Authors' contributions}

CL initiated the research. ZW and CL carried out the experiments and analyzed the data. Both authors drafted the manuscript, then reviewed and approved the final version.

\section{Ethics approval and consent to participate}

The present study was approved by the Ethics Committee of the First Affiliated Hospital of Jinzhou Medical University (Jinzhou, China). Written informed consent was signed by all patients.

\section{Patient consent for publication}

Not applicable. 


\section{Competing interests}

The authors declare that they have no competing interests.

\section{References}

1. Graham DY. Helicobacter pylori update: gastric cancer, reliable therapy, and possible benefits. Gastroenterology. 148: 719-731, 2015.

2. Allemani C, Weir HK and Carreira H, et al. Global surveillance of cancer survival 1995-2009: analysis of individual data for $25,676,887$ patients from 279 population-based registries in 67 countries (CONCORD-2). Lancet. 385: 977-1010, 2015.

3. Orditura M, Galizia G and Sforza V, et al. Treatment of gastric cancer. World J Gastroenterol. 20(7): 1635-1649, 2014.

4. Park $Y$, et al. The effectiveness and safety of endoscopic submucosal dissection compared with endoscopic mucosal resection for early gastric cancer: a systematic review and metaanalysis. Surg Endosc. 25(8): 2666-2677, 2011.

5. Choi et al. Long-term outcomes of endoscopic submucosal dissection for early gastric cancer: a single-center experience. Surg Endosc. 27(11): 4250-4258, 2013.

6. Memczak $S$ et al. Circular RNAs are a large class of animal RNAs with regulatory potency. Nature.495: 333-338, 2013.

7. Salzman J, Gawad C, Wang P, Lacayo N, Brown P. Circular RNAs are the predominant transcript isoform from hundreds of human genes in diverse cell types. Plos One,7, e30733, 2012.

8. Conn S. et al. The RNA binding protein quaking regulates formation of circRNAs. Cell.160: 11251134, 2015.

9. Vicens Q andWesthof E. Biogenesis of Circular RNAs. Cell.159: 13-14, 2014.

10. Hansen Tet al. Natural RNA circles function as efficient microRNA sponges. Nature.495: 384-388, 2013.

11. Yao Z, Luo J, Hu K et al. ZKSCAN1 gene and its related circular RNA (circZKSCAN1) both inhibit hepatocellular carcinoma cell growth, migration, and invasion but through different signaling pathways. Mol Oncol. 11(4):422-437, 2017.

12. Li J and Song Y. Circular RNA GLI2 promotes osteosarcoma cell proliferation, migration, and invasion by targeting miR-125b-5p. Tumour Biol. 39(7), 2017.

13. You $X$ et al. Neural circular RNAs are derived from synaptic genes and regulated by development and plasticity. Nat Neurosci.18: 603-610, 2015.

14. Sun $\mathrm{H}$ et al. Circ-SFMBT2 promotes the proliferation of gastric cancer cells through sponging miR182-5p to enhance CREB1 expression. Cancer Manag Res. 16 (10): 5725-5734, 2018.

15. Zhang Y, Liu H, Li W, et al. CircRNA_100269 is downregulated in gastric cancer and suppresses tumor cell growth by targeting miR-630. Aging. 9(6): 1585-1593, 2017. 
16. Feng J, Wang X, Zhu W, Chen S, Feng C. MicroRNA-630 suppresses Epithelial-to-Mesenchymal Transition by regulating FoxM1 in gastric cancer cells. Biochemistry (Mosc). 82(6): 707-714, 2017.

17. Sun Y, et al. MiR-630 inhibits Endothelial-Mesenchymal Transition by targeting slug in traumatic heterotopic ossification. Scientific reports. 6: 2272-2285, 2016.

18. Yang L, Liu Y, Wang M, Qian Y, Dai X, Zhu Y, Chen J, Guo S, Hisamitsu T: Celastrus orbiculatus extract triggers apoptosis and autophagy via PI3K/Akt/mTOR inhibition in human osteosarcoma cells. Oncol Lett. 12: 3771-3778, 2016.

19. He F, Chen H, Yang P, Wu Q, Zhang T, Wang C, Wei J, Chen Z, Hu H, Li W, Cao J: Gankyrin sustains $\mathrm{PI3K} / \mathrm{GSK}-3 \mathrm{beta} /$ beta-catenin signal activation and promotes osteosarcoma aggressiveness and progression. Oncotarget. 7:81156-81171, 2016.

20. Fan X, Wang Y, Wang L, Zhu M: Salidroside induces apoptosis and autophagy in human osteosarcoma cells through inhibition of PI3K/Akt/mTOR pathway. Oncol Rep. 36: 3559-3567, 2016.

21. Qian D, Xiao X, Byun J, Suriawinata A, Her S, Amos C, Barth R: PI3K/Akt/mTOR signaling and plasma membrane proteins are implicated in responsiveness to adjuvant dendritic cell vaccination for metastatic osteosarcoma. Clin Cancer Res. 23: 399-406, 2017.

22. Fang $X$, Wen $J$ and Sun $M$, et al. CircRNAs and its relationship with gastric cancer. J Cancer. 10(24): 6105-6113, 2019.

23. Wang Z, Ma K and Pitts S, et al. Novel circular RNA circNF1acts as a molecular sponge, promoting gastric cancer by absorbing miR-16. Endocrine-Related Cancer. 3(26): 265-277, 2019.

24. Shi P, Wan J, Song H and Ding X. The emerging role of circular RNAs in gastric cancer. Am J Cancer Res. 8(10): 1919-1932, 2018.

25. Wang K and Dong M. Role of circular RNAs in gastric cancer: Recent advances and prospects. World J Gastrointest Oncol. 11(6): 459-469, 2019.

26. Zhu Z, Rong Z and Luo Z, et al. Circular RNA circNHSL1 promotes gastric cancer progression through the miR-1306-3p/SIX1/vimentin axis. Molecular Cancer. 18, 126-135, 2019.

27. Shan $C$, Zhang $Y$ and Hao $X$, et al. Biogenesis, functions and clinical significance of circRNAs in gastric cancer. Molecular Cancer. 18, 136-145, 2019.

28. Alexander W: Inhibiting the Akt pathway in cancer treatment. Pharmacy and therapeutics, 36: 225227, 2011.

29. Nitulescu G, Margina D and Tsatsakis A, et al.: Akt inhibitors in cancer treatment: The long journey from drug discovery to clonical use. Int J Oncol, 48: 869-885, 2015.

30. Brown $\mathrm{J}$ and Banerji U: Maximizing the potential of Akt inhibitors as anti-cancer treatments, Pharmocol Ther, 172: 101-115, 2017.

31. Jansen V, Mayer I and Arteaga C: Is there a future for Akt inhibitors in the treatment of cancer? 22: 2599-2601, 2016.

32. Mayer I and Arteaga C: The PI3/Akt pathway as a target for cancer treatment. Annual review of medicine, 67: 11-28, 2016. 
33. Suvarna V, Murahari M and Khan T: Phytochemicals and PI3K inhibitors in cancer-an insight. Front. Pharmacol., 8:1-23, 2017.

\section{Table}

Table I. Clinical and pathological characteristics of patients with GC enrolled in the present study.

\begin{tabular}{|c|c|c|c|c|}
\hline \multirow[t]{2}{*}{ Parameters } & \multirow[t]{2}{*}{$\mathrm{n}$} & circRNA_100269 & expression & \multirow[t]{2}{*}{$P$ value } \\
\hline & & Low & High & \\
\hline Gender & & & & 0.464 \\
\hline Male & 26 & 12 & 14 & \\
\hline Female & 30 & 16 & 14 & \\
\hline Age (years) & & & & 0.429 \\
\hline$>60$ & 24 & 11 & 13 & \\
\hline$\leq 60$ & 32 & 17 & 15 & \\
\hline Tumor size $(\mathrm{cm})$ & & & & 0.524 \\
\hline$>5$ & 24 & 12 & 12 & \\
\hline$\leq 5$ & 32 & 16 & 16 & \\
\hline Histology grade & & & & $0.023^{*}$ \\
\hline I-II & 34 & 13 & 21 & \\
\hline III-IV & 22 & 15 & 7 & \\
\hline Smoking & & & & 0.485 \\
\hline Yes & 31 & 16 & 15 & \\
\hline No & 25 & 12 & 13 & \\
\hline Metastasis & & & & $0.019^{*}$ \\
\hline Yes & 22 & 15 & 7 & \\
\hline No & 34 & 13 & 21 & \\
\hline
\end{tabular}

Differences among variable were analyzed using the $\chi 2$ test, * indicates that the values have statistically significant differences.

\section{Figures}


A

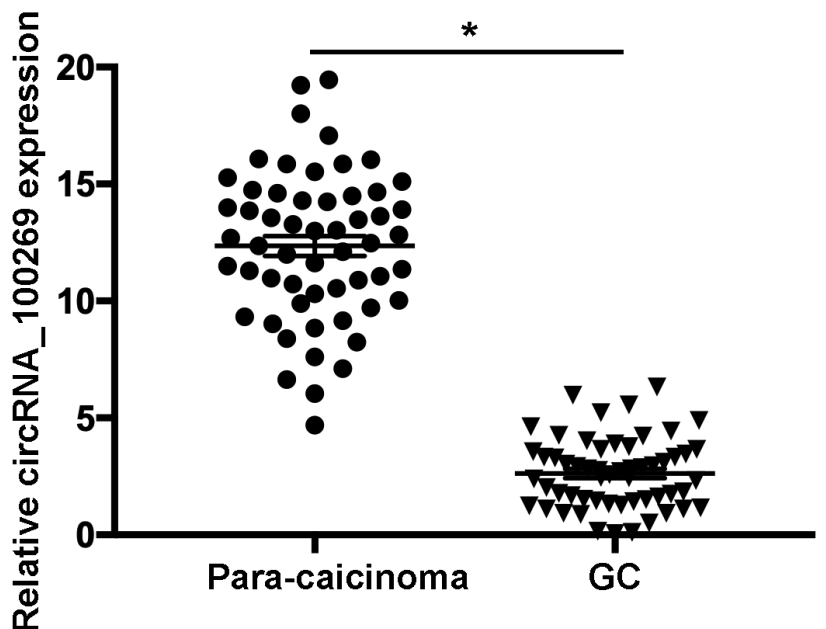

C $\subsetneq$

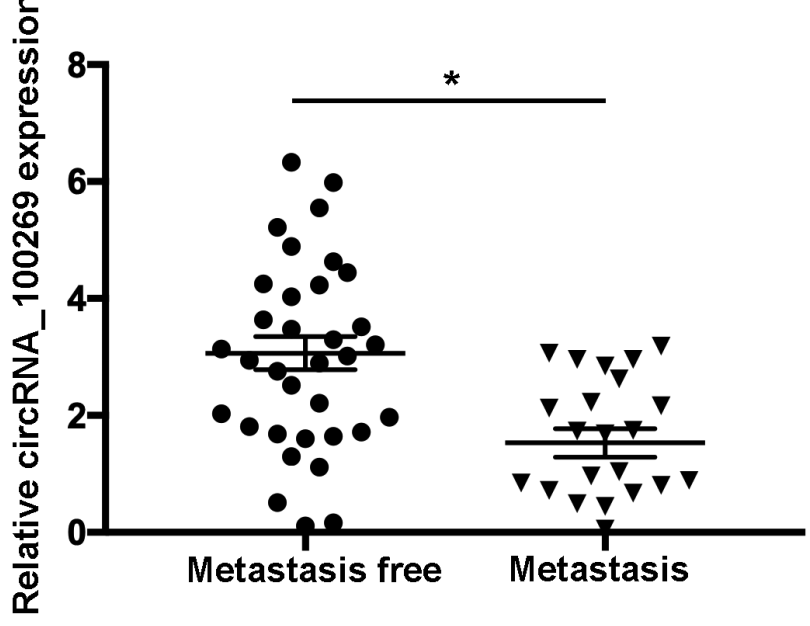

B
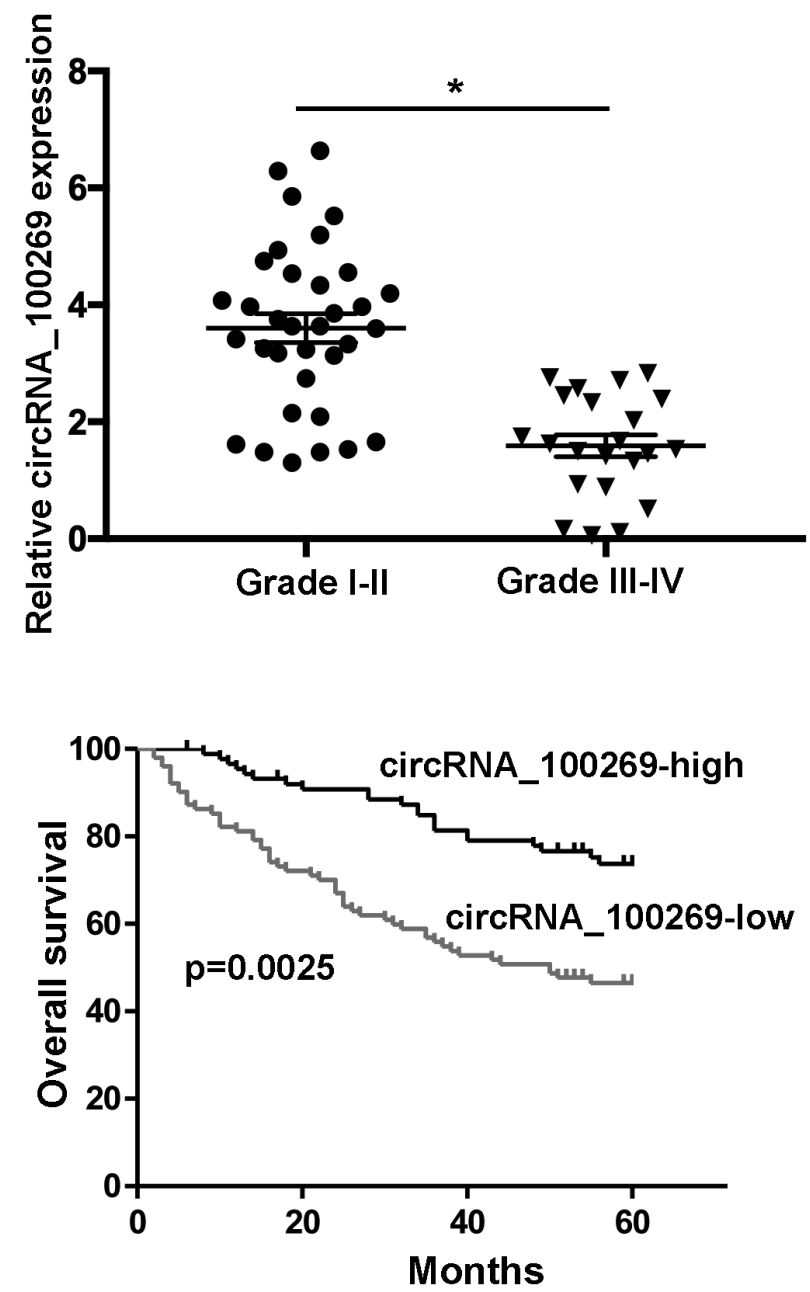

E

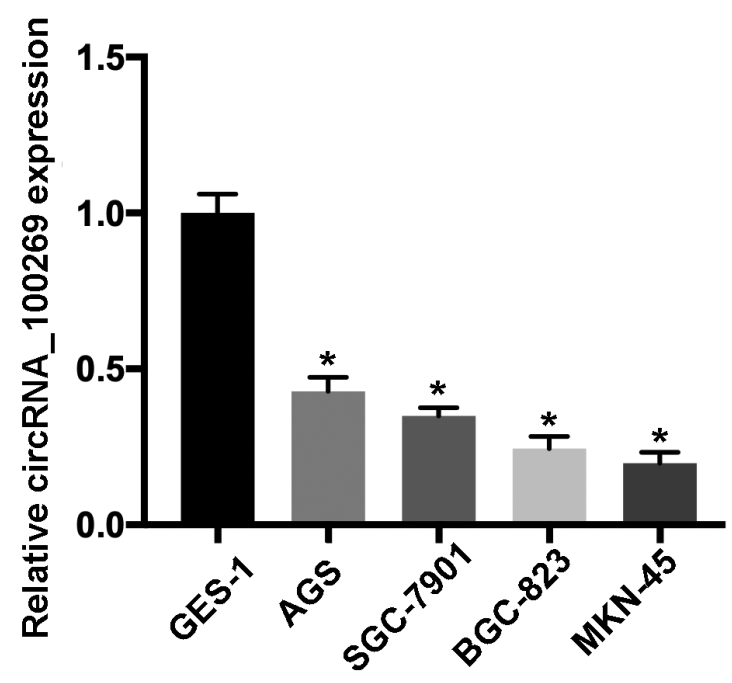

Figure 1

The expression of circRNA_100269 is downregulated in GC tissues and cells. (A) The level of circRNA_100269 was determined in 56 GC tissues and paired non-tumor samples using reverse transcription-quantitative polymerase chain reaction. (B) CircRNA_100269 expression was assessed in GC patients with different tumor grades. (C) The level of circRNA_100269 was evaluated in GC tissues with or without metastasis. (D) Survival analysis of GC patients with low- or high- circRNA_100269 
expression. (E) The expression of circRNA_100269 was examined in GC cell lines (AGS, SGC-7901, BGC823 and MKN-45) and one normal human gastric epithelial cell line (GES-1). ${ }^{*} \mathrm{P}<0.05$. GC, gastric cancer.

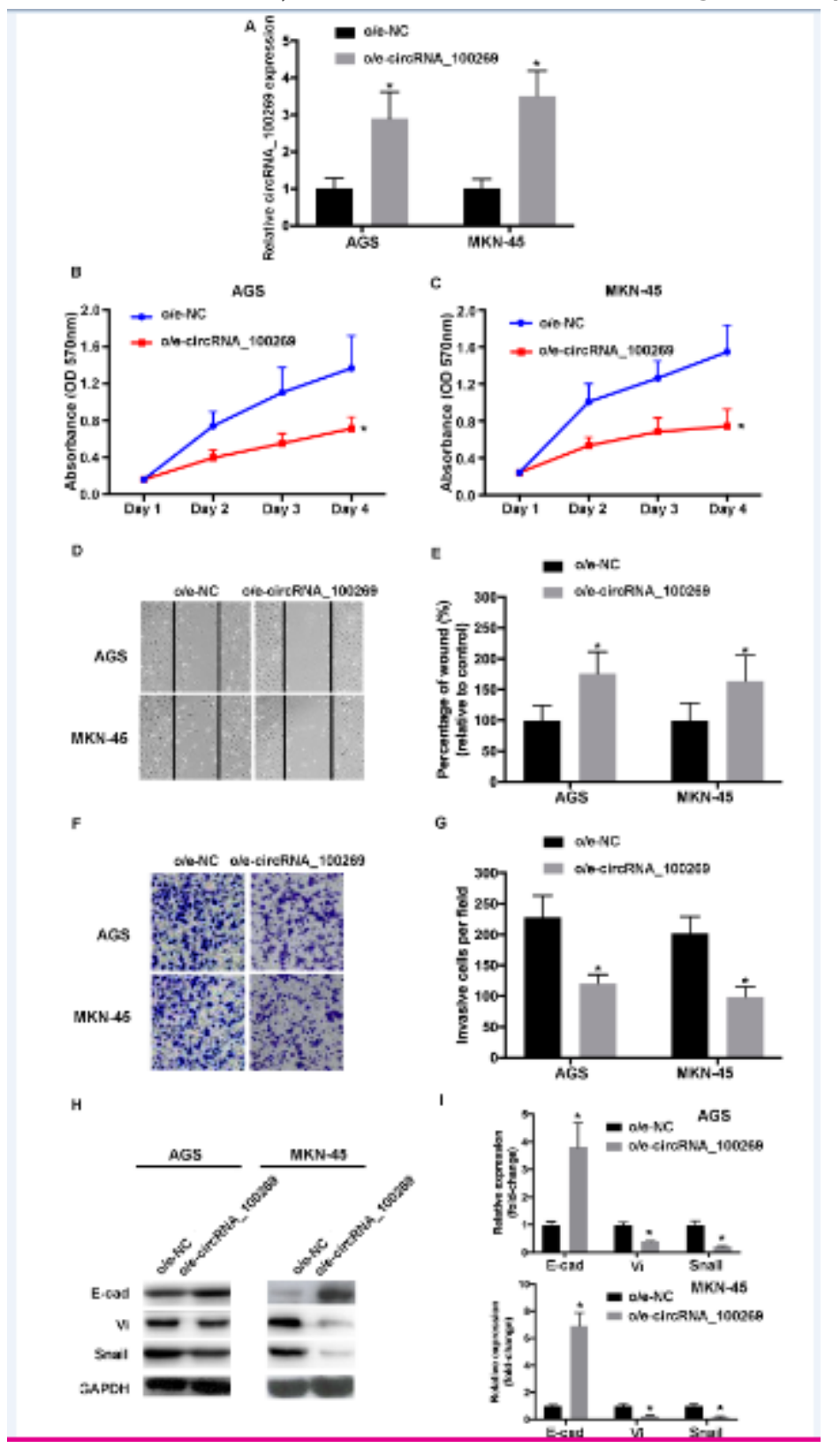

Figure 2

Upregulated circRNA_100269 inhibits the proliferation, migration, invasion and EMT of GC cells. (A) Transfection efficiency of o/e-circRNA_100269 was confirmed using RT-qPCR. (B and C) The proliferation of GC cells transfected with o/e-circRNA_100269 or o/e-NC were determined using Cell Counting Kit-8 assay. (D and $E$ ) The migration of transfected AGS and MKN-45 cells were evaluated using a wound healing assay (magnificationx100). ( $F$ and G) The invasive activity of GC cells transfected with o/ecircRNA_100269 or o/e-NC were examined (magnificationx200). (H and I) The expression levels of EMTassociated markers were evaluated using RT-qPCR and western blotting. ${ }^{*}<0.05$. GC, gastric cancer; NC, negative control; RT-qPCR, reverse transcription-quantitative polymerase chain reaction. 
A

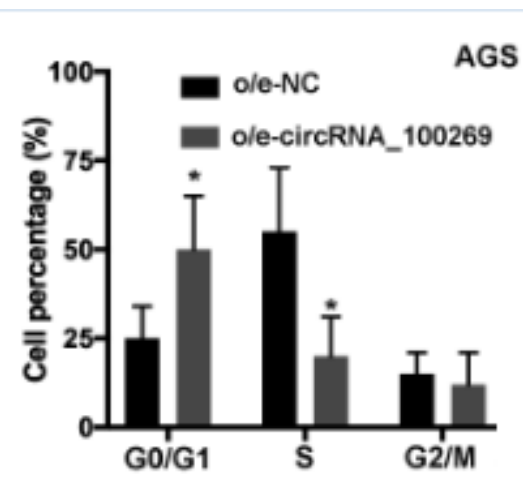

C

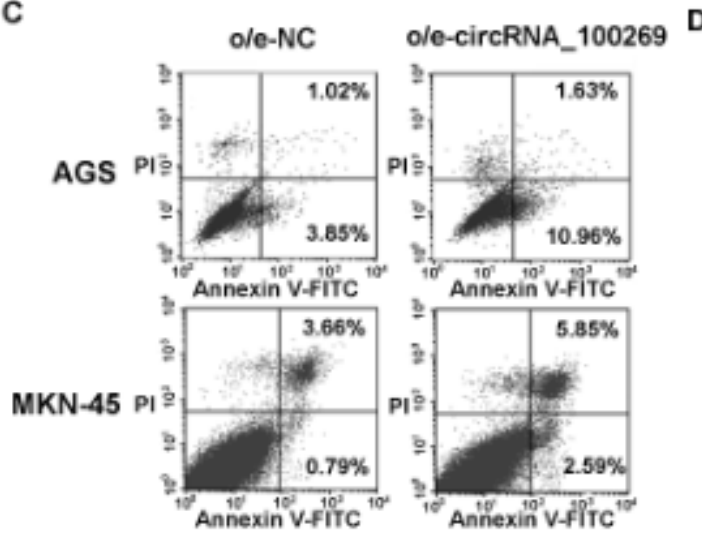

B

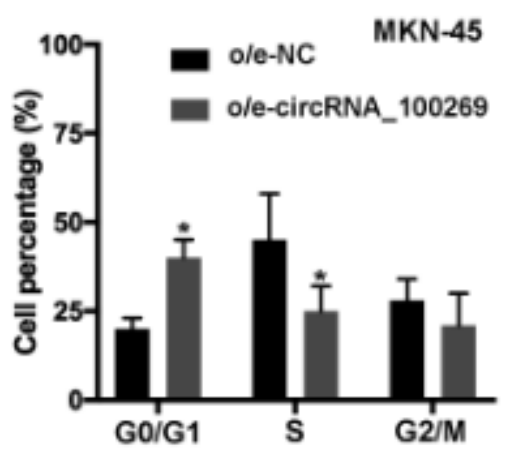

D

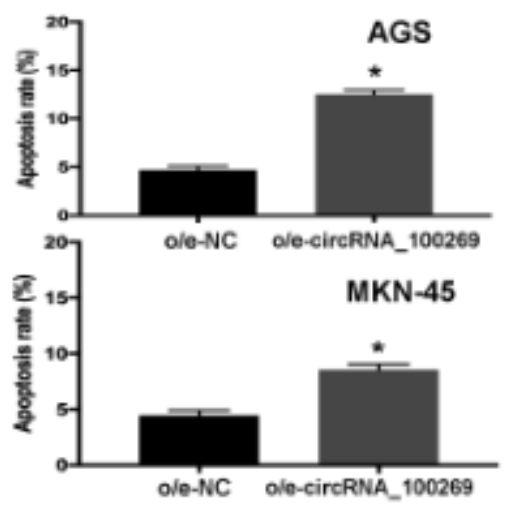

E

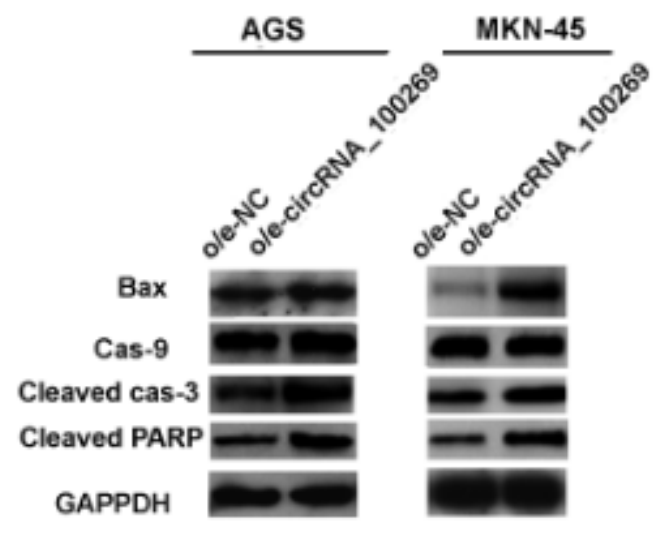

\section{Figure 3}

Overexpression of circRNA_100269 induces cell cycle arrest and apoptosis in GC cells. (A and B) The distribution of cell cycle and apoptosis in GC cells overexpressing circRNA_100269 were examined. (C and D) The apoptosis of AGS and MKN-45 cells transfected with o/e-circRNA_100269 were also determined using flow cytometry. (E) The expression levels of apoptosis-associated markers were evaluated in transfected GC cells compared with the control. ${ }^{*} \mathrm{P}<0.05$. GC, gastric cancer. 


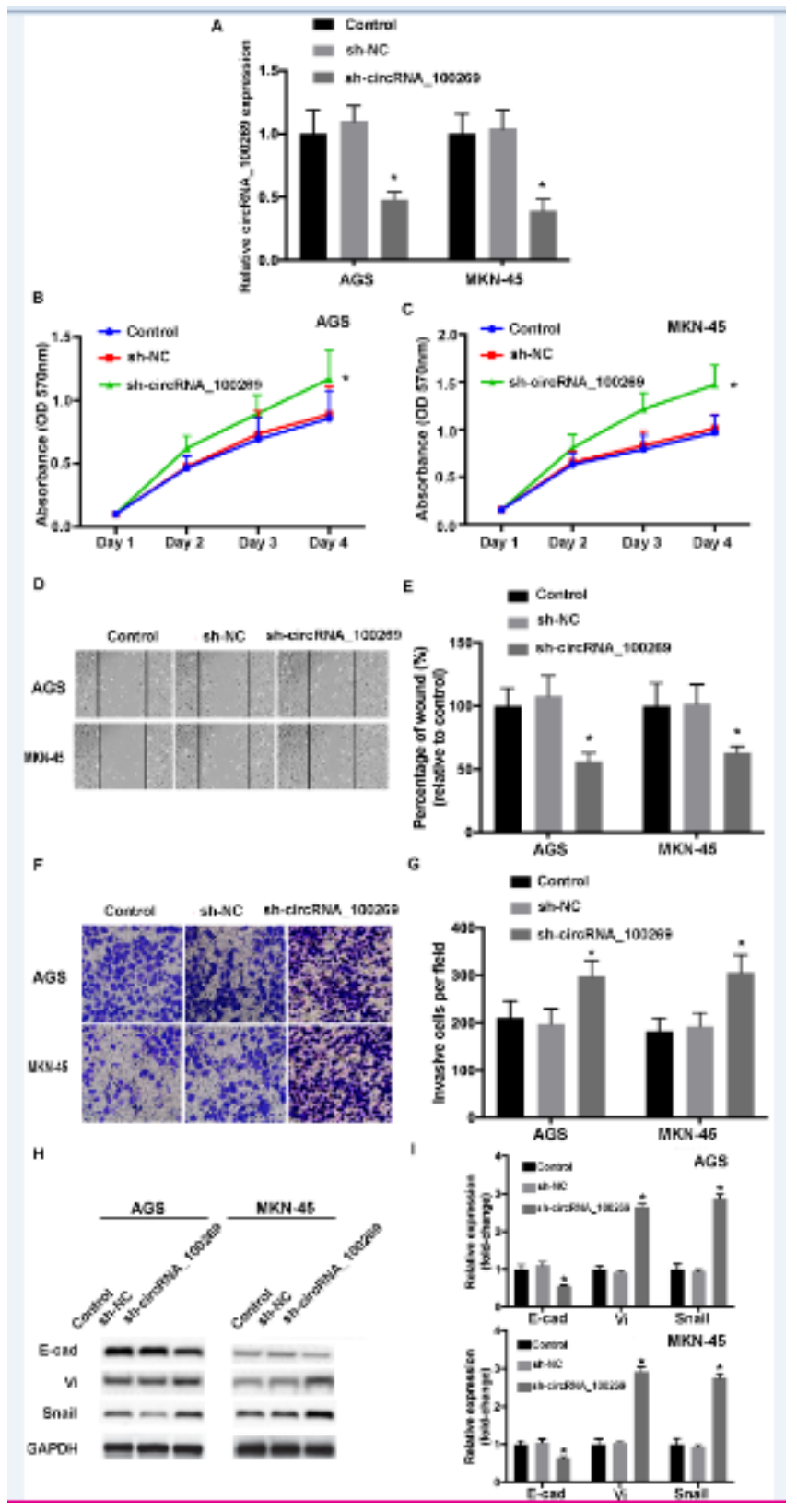

Figure 4

Knockdown of circRNA_100269 promotes the proliferation, migration, invasion and EMT of GC cells. (A) Transfection efficiency of sh-circRNA_100269 was evaluated by RT-qPCR. (B and C) The viabilities of GC cells transfected with sh-circRNA_100269 or sh-NC were examined using Cell Counting Kit-8 assay. (D-G) The migration and invasion of transfected AGS and MKN-45 cells were determined using wound healing (magnificationx100) and Transwell assay (magnificationx200). ( $\mathrm{H}$ and I) The levels of EMT-related molecules were determined using RT-qPCR and western blotting. ${ }^{*}<0.05$ vs. control group. GC, gastric cancer; NC, negative control; RT-qPCR, reverse transcription-quantitative polymerase chain reaction. 


$$
\text { A }
$$

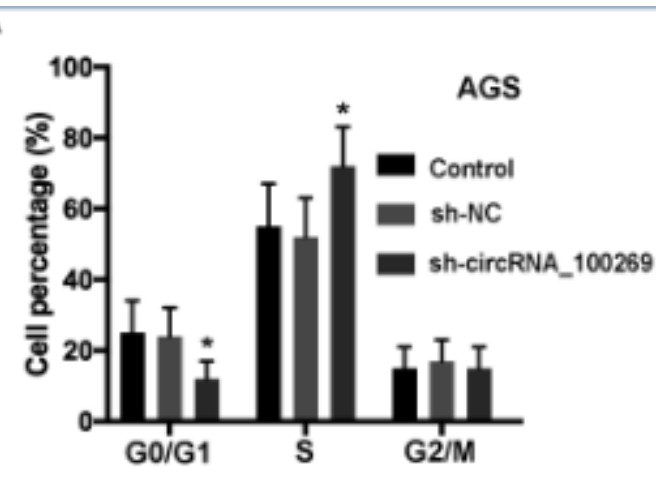

C

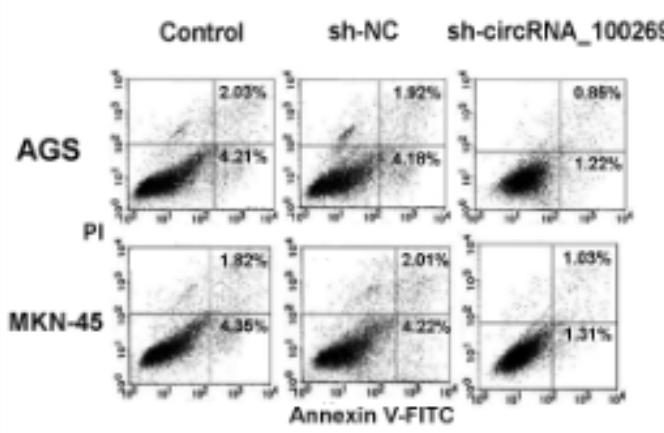

E

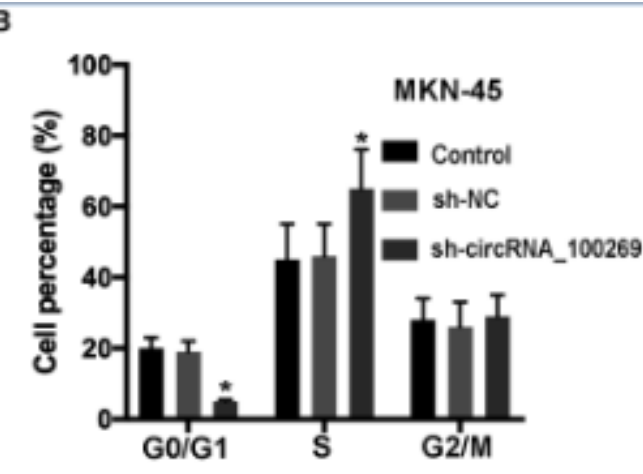

D
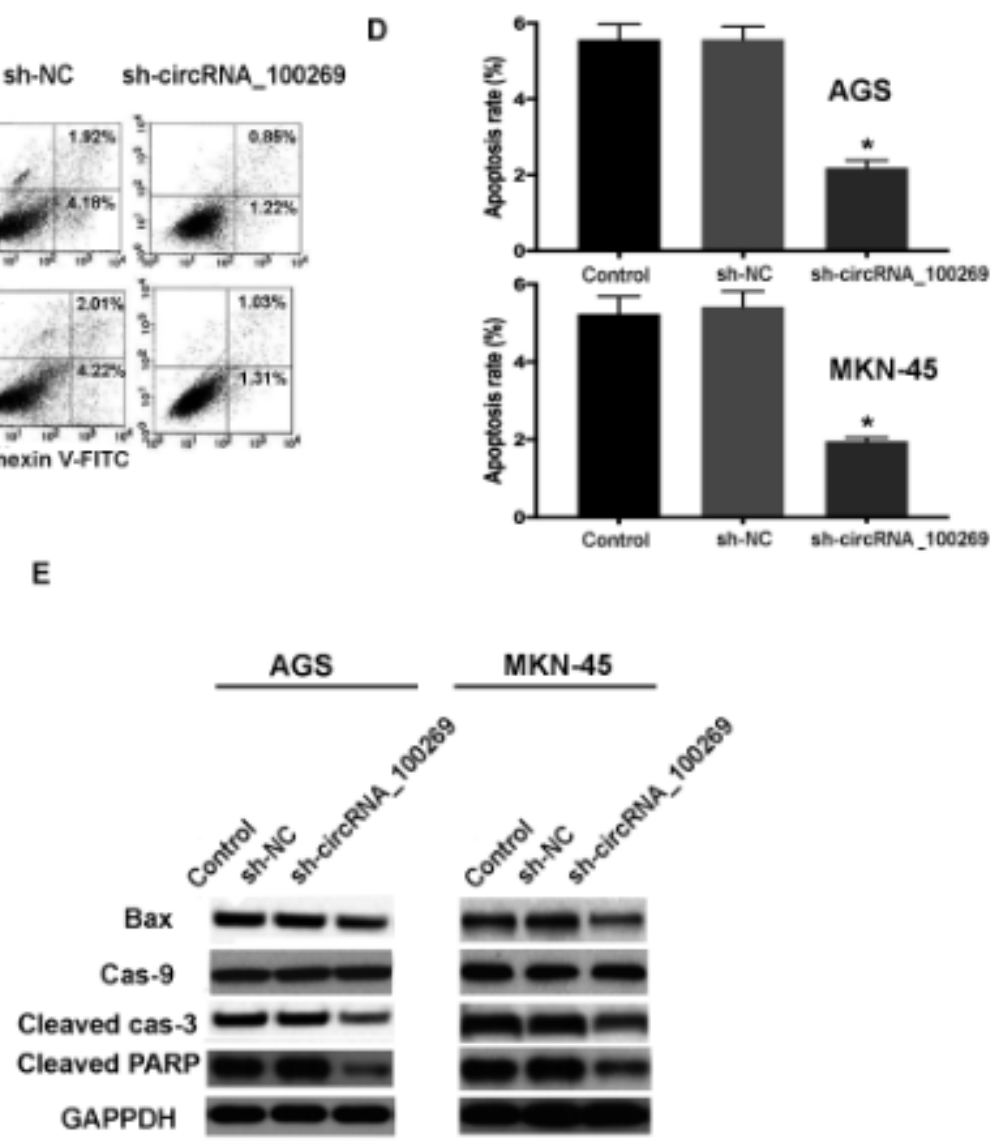

Figure 5

Knockdown of circRNA_100269 inhibits cell cycle arrest and apoptosis of GC cells. (A and B) The distribution of cell cycle and apoptosis in GC cells transfected with sh-circRNA_100269 were determined. (C-E) The apoptosis of AGS and MKN-45 cells with circRNA_100269 knockdown were also examined compared with the controls. ${ }^{*} \mathrm{P}<0.05$ vs. control group. GC, gastric cancer. 


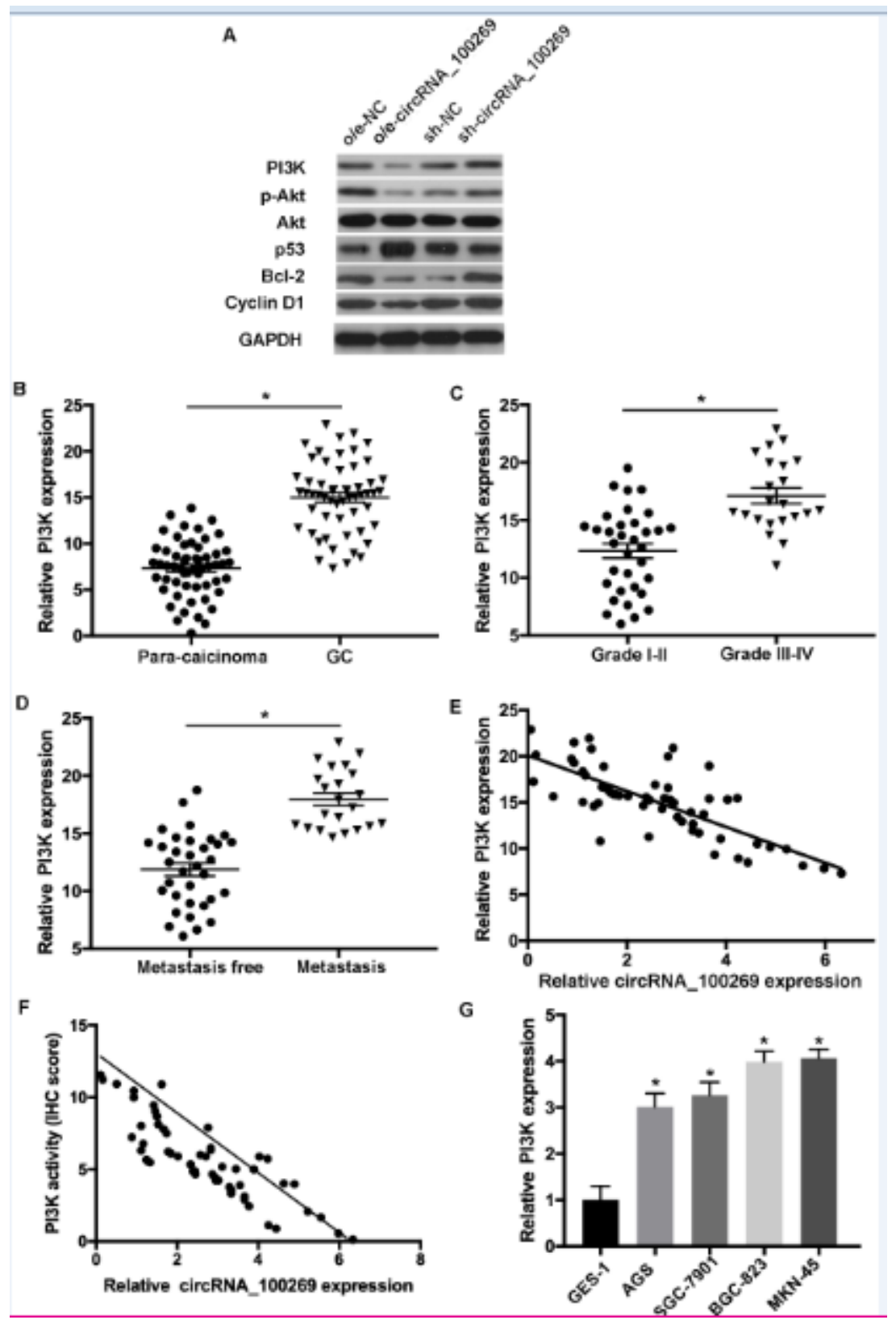

Figure 6

$\mathrm{PI3K} /$ Akt signaling is the promising target of circRNA_100269 in GC cells. (A) The levels of molecules involved in PI3K/Akt signaling were assessed in AGS and MKN-45 cells with overexpression/knockdown of circRNA_100269. (B) The expression of PI3K was examined in GC and matched non-tumor tissues. (C) $\mathrm{PI} 3 \mathrm{~K}$ expression was evaluated in GC patients with various tumor grades. (D) The expression level of $\mathrm{PI} 3 \mathrm{~K}$ was determined in GC tissues with metastasis compared to the controls. (E) Spearman's correlation analysis indicated the inverse correlation between circRNA_100269 and PI3K in GC samples ( $r=-0.3291$; $P=0.00938)$. ( $F)$ The activity of PI3K was negatively correlated with the expression of circRNA_100269 in GC specimens ( $r=-0.6211 ; P=0.00812)$. ( $G)$ The level of PI3K was also assessed in $G C$ cells compared with normal human gastric epithelial cells. ${ }^{*} \mathrm{P}<0.05$. GC, colorectal cancer. 


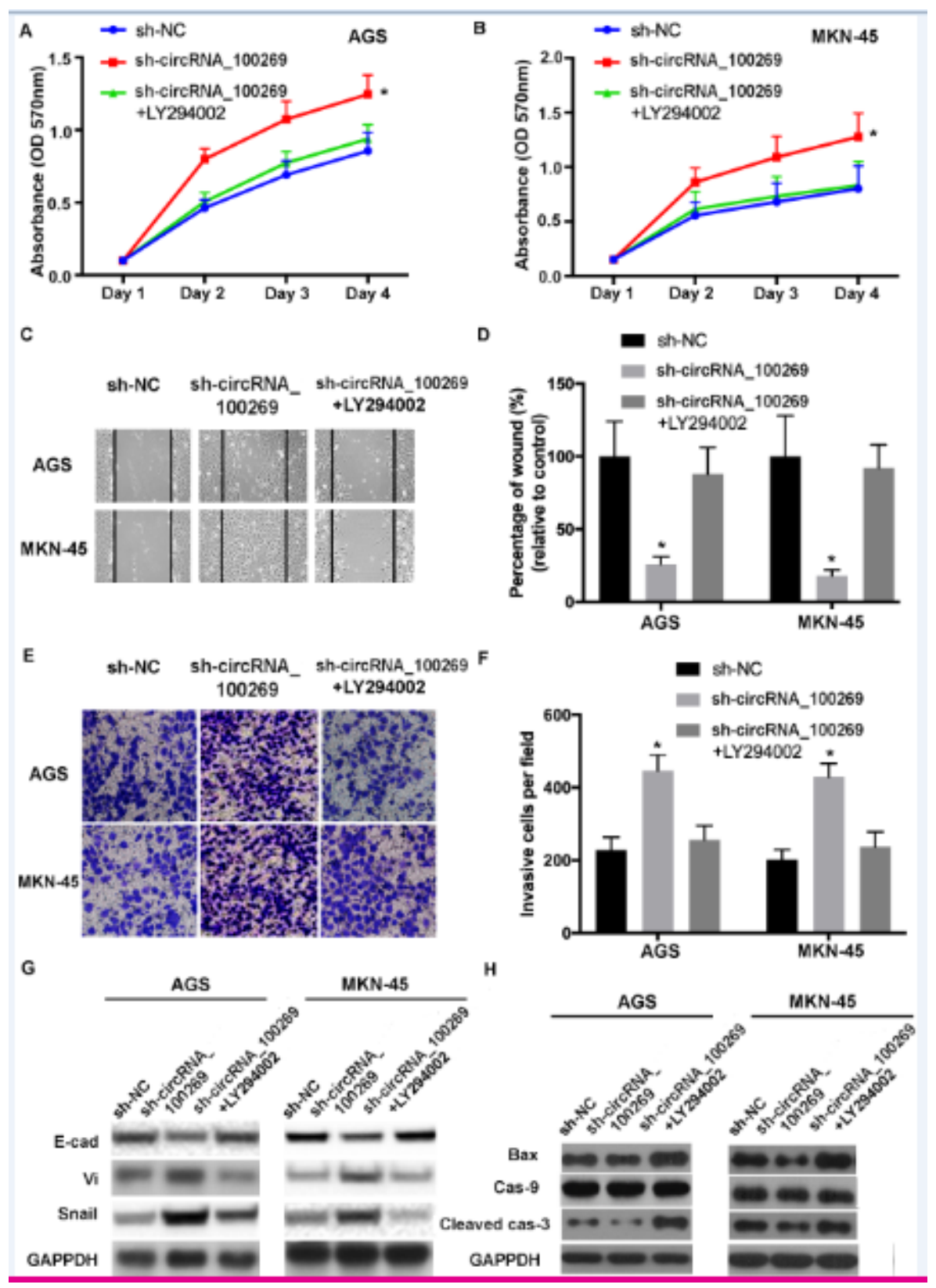

Figure 7

Inactivation of PI3K/Akt signaling reverses the effects of circRNA_100269 knockdown in GC cells. (A and B) The proliferation of GC cells transfected with sh-NC, sh-circRNA_100269 or co-treated with LY294002 was assessed. (C-F) The migration and invasion of treated AGS and MKN-45 cells were determined. (G and H) EMT and apoptosis of transfected GC cells were evaluated. ${ }^{*} \mathrm{P}<0.05$ vs. sh-NC. GC, gastric cancer; NC, negative control. 
A

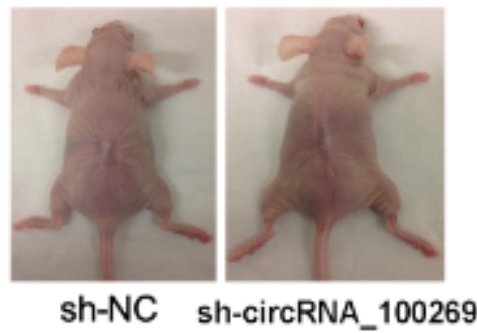

sh-NC sh-circRNA_100269

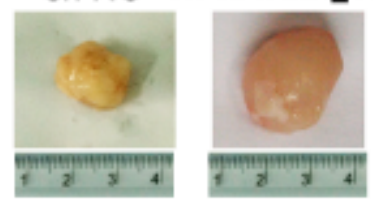

B

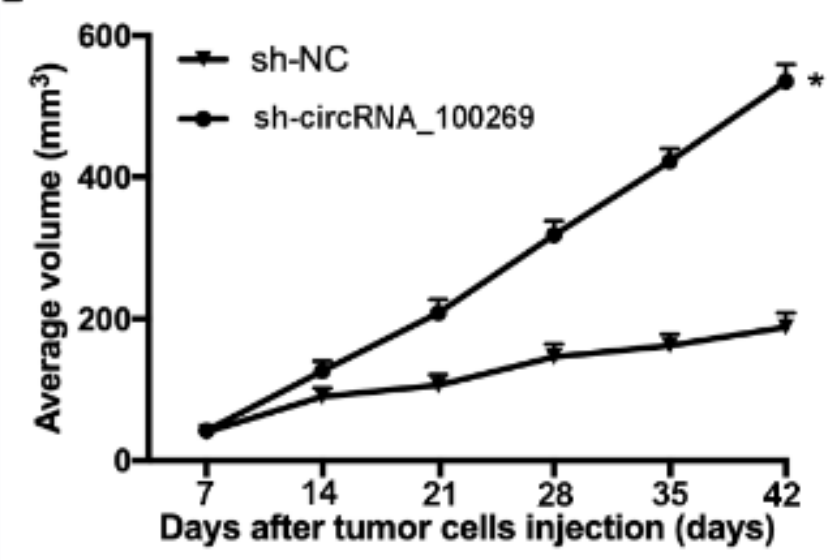

D

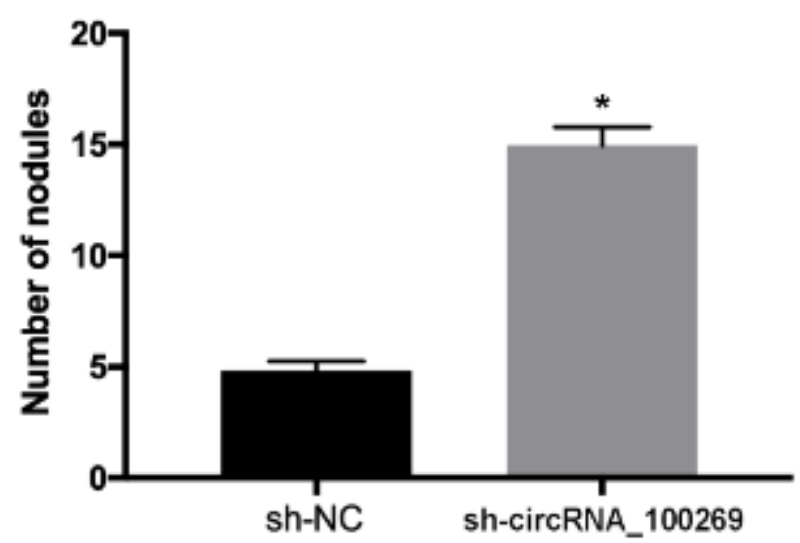

C

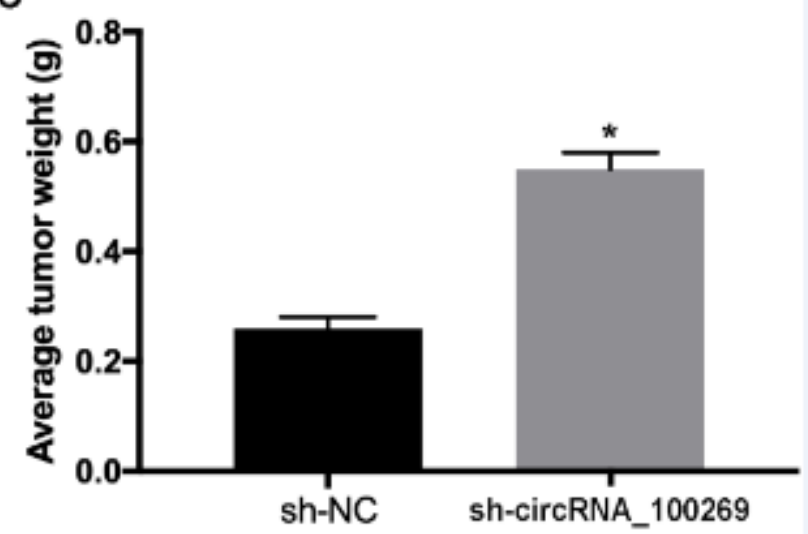

E

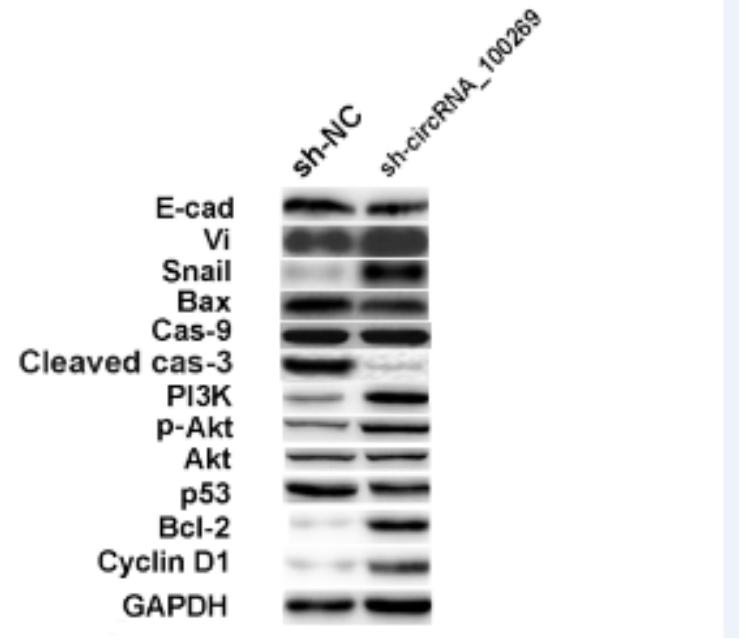

Figure 8

Downregulated circRNA_100269 promotes the growth of GC in vivo. (A) The tumors in mice were harvested six weeks post-injection. (B) The average tumor volumes were examined in sh-circRNA_100269 group compared with the control. (C) Orthotopic tumor weights at day 42 post-injection were determined. (D) The numbers of nodules in mice were also calculated six weeks post-injection. (E) mRNA levels of EMT, apoptosis and PI3K/Akt-associated molecules in xenograft tumors were evaluated by western blot analysis. A total of five mice were included in each experimental group. ${ }^{\star} \mathrm{P}<0.05 . \mathrm{n}=5$ per each group. GC, gastric cancer. 